\title{
Time Trends And Age-Period-Cohort Effects On The Incidence Of Gastric Cancer In Changle From 2003 To 2012
}

Yongtian $\operatorname{Lin}^{1}, *$

Shaowei $\operatorname{Lin}^{2, *}$

Jianshun Chen ${ }^{3}$

Lici Chen ${ }^{3}$

Tao Tao ${ }^{2}$

Siying $\mathrm{Wu}^{2}$

'Department of Epidemiology, Fujian Cancer Hospital and Fujian Medical University Cancer Hospital, Fu Zhou, People's Republic of China; ${ }^{2}$ Department of Epidemiology and Health Statistics, School of Public Health, Fujian Medical University, Fu Zhou, People's Republic of China; ${ }^{3}$ Department of Office, Changle Cancer Research Institute, Fu Zhou, People's Republic of China

*These authors contributed equally to this work
This article was published in the following Dove Press journal: Cancer Management and Research

Purpose: Although the incidence of gastric cancer in China has declined over the past decades, they were still much higher than the average of global. This aim of this study was to describe the trends and age-period-cohort effects on gastric cancer incidence from 2003 to 2012 in Changle and to explore the potential reason.

Materials and methods: Data on patients with gastric cancer diagnosed between 2003 and 2012 were collected by the population-based Changle cancer registration $(n=4111)$. Agestandardized incidence rates of gastric cancer were calculated and joinpoint regression was used to evaluate the trends of gastric cancer incidence. Time trends in gastric cancer incidence by the period of diagnosis and birth cohort were analyzed by sex. Age-periodcohort analysis was performed to investigate the independent effects of age, period of diagnosis and birth cohort among over 25 -year-old residents.

Results: A steady downward trend was observed among men, with the incidence ranging from 96.15 per 100,000 in 2003 to 62.6 per 100,000 in 2012 (APC, $-5.1 \%$; $95 \%$ CI: -6.9 to $-3.2 \%$ ). A similarly declining trend was observed among women with the incidence ranging from 34.5 per 100,000 to 15.7 per 100,000 (APC, $-5.7 \%$; $95 \%$ CI: -9.3 to $-2.0 \%$ ). Age-period-cohort model of incidence rate showed increasing age effect and decreasing period of diagnosis effects in both men and women. Birth cohorts exhibited a decreasing trend in the incidence among women who were born after 1935 and men after 1940.

Conclusion: Recent decreases in the incidence of gastric cancer were due to decreased period of diagnosis and cohort effects, which was attributed to the improvements in their lifestyle and habits.

Keywords: gastric cancer, incidence, trend, age-period-cohort model

\section{Introduction}

Gastric cancer remains one of the most common cancers worldwide. As estimated, almost 10 million new cases of gastric cancer occurred in 2018 (5.7\% of the total cancer cases), accounting for the fifth most common cancer globally. ${ }^{1}$ In the past, more than $70 \%$ of cases were estimated to occur in developing countries. Among these cases, nearly half were distributed in Eastern Asia in 2012 (mainly in China). ${ }^{2}$ In China, 76,243 new cases of gastric cancer (incidence: 33.7 per 100,000) were reported, ranking third to lung cancer and breast cancer in $2013 .^{3}$ Gastric cancer remains a huge challenge to the health of the Chinese population.

Notable regional distribution characteristics of gastric cancer mainly concentrated in the eastern and southwestern regions (52.2\%) of China. ${ }^{4}$ Changle, located
Department of Epidemiology and Health Statistics, School of Public Health, Fujian Medical University, No. I Xuefu North

Road, University Town, Fu Zhou 350I22, People's Republic of China

Tel +8659122862510

Fax +8659188783929

Emailfmuwsy@163.com 
on the southeast coast of Fujian Province, China, and with a total population of 700,000 , represents a high-incidencerate area of gastric cancer, with the mortality rate of gastric cancer reaching 120.5 per 100,000 . The mortality rate among Changle men was five-folds higher than the national average level $\left(20.9\right.$ per 100,000) in the 1970s. ${ }^{5}$ The population-based cancer registration system in Changle was established in 1988, when gastric cancer still ranked the first in the incidence of cancer in Changle. ${ }^{6}$

A definite decreasing trend of gastric cancer was observed recently in developed countries; this phenomenon was also observed in Changle residents in 2013 and 2014. ${ }^{6-8}$ Important factors, such as Helicobacter pylori (HP) infection, lifestyle, and largely improved dietary habits among Changle residents in the past 20 or 30 years, might reduce the prevalence of gastric cancer in Changle. ${ }^{5,-11}$ Previous studies on the incidence of gastric cancer in Changle had declined in different periods since 1988. ${ }^{12,13}$ These studies, which used the traditional analysis method, showed limited power in explaining how the incidence rate at different periods was affected by age and birth cohort. Age-period-cohort analysis would help in investigating the independent effects of age, period of diagnosis, and birth cohort on the incidence trend of gastric cancer. ${ }^{14}$

Therefore, the present study aimed to describe secular trends in the incidence rate of gastric cancer in Changle and to evaluate the independent effects of age, period of diagnosis, and birth cohort by using age-period-cohort analysis.

\section{Materials And Methods Data Sources}

The population-based Changle cancer registries collected information about cancer cases from 1988. Changle cancer registry was a monitoring site in Fujian Province, with the established longest period of cancer data registration. The registration data of the whole population in Changle showed relatively high quality and accuracy, which were cited by incidence and mortality of cancer in some cities and counties of China (I-IV), Chinese cancer registry annual report (2008-2016), and Cancer Incidence in Five Continents (VIII).

Registered gastric cancer cases were coded according to the code of $\mathrm{C} 16$ from the tenth Revision of the International Classification of Disease. Information, including name, sex, age, date of birth, year of diagnosis, cancer site, was collected by Changle Cancer Research Institute. Identifiable information, such as patient's name, ID number, telephone number, and detailed address, had been deleted in the database provided by the Changle Cancer Research Institute in this study. We used midyear population data as the total population in the year to evaluate the incidence rate of gastric cancer. The population data during the corresponding period of diagnosis were obtained from Changle Police Department.

This study was approved by the Institute Medical Ethics Committee of Fujian Cancer Hospital (KT2019010-01) and conducted according to the Declaration of Helsinki. The requirement for informed consent was waived because of the retrospective nature of this study, but the patient's identifiable information was protected.

\section{Quality Control}

Cancer registration was conducted according to the quality control requirements of Chinese Guideline for Cancer Registration. The proportion of morphological verification (MV\%) reached 66-85\%, the percentage of cancer cases identified with death certification only (DCO\%) totaled 0 $10 \%$, and mortality-to-incidence ratio (M/I) spanned 0.60.8 . The $\mathrm{MV} \%$ of incidence rate of gastric cancer was $62.0 \%$ (2549/4111), DCO\%, 0.9\% (37/4111), and M/I, $0.8(3083 / 4111)$ in 2003-2012. All the above findings satisfied the quality requirements of cancer registration in China.

\section{Statistical Analysis}

The population data used for evaluating the incidence of gastric cancer in Changle were derived from the database of Changle cancer registry between 2003 and 2012. New gastric cancer cases were allocated to a period according to the year of diagnosis and to a birth cohort according to their year of birth. The age-standardized incidence rates were calculated per 100,000 by the direct method by using Segi 1960 world standard population. ${ }^{15}$ We estimated the tendency of age-standardized incidence rates by employing the joinpoint regression analysis program with the results expressed as annual percent changes (APC) and 95\% confidence intervals (CI). ${ }^{16}$ The statistical analyses were performed using Joinpoint software ver. 4.6.0.0 (Surveillance Research Program, National Cancer Institute, Bethesda, MD).

Incidence trends were evaluated according to sex by using Poisson regression with logarithmic offset by utilizing maximum likelihood estimation, with the trends described by gender, age, and birth cohort. The expression 
of the age-period-cohort analysis model (APC model) is $Y$ $=\alpha_{0}+\alpha X_{1}+\beta X_{2}+\gamma X_{3}+\varepsilon$. In this study, $Y$ denotes gastric cancer incidence, $X_{1}, X_{2}$, and $X_{3}$ refer to the age, period, and birth cohort, respectively. $\alpha_{0}$ is the intercept, and $\varepsilon$ is the residual. The effects of $\alpha, \beta$, and $\gamma$ are parameters to be estimated. Birth cohort $=$ period - age. The incidence rates of gastric cancer were categorized into 13 age groups (25$29,30-34,35-39, \ldots$, and 85 years or older), in which we excluded those under 25 years old because of the small numbers of cases, which accounted for $0.3 \%$ of the participants. The study period was 2003-2012, the oldest age group was over 85 years old, and the corresponding birth cohort was 1918-1922. By analogy, the age group was 2529 years old, and the corresponding birth cohort was the latest, that is, 1983-1987. The Poisson logarithmic linear model was used to solve the APC model. Logarithmic linear transformation was performed on both sides of the above equation. The expression of transformation is as follows: $\ln \left[E\left(M_{i j}\right)\right]=\ln \left(D_{i j} / P_{i j}\right)=\mu+\alpha_{i}+\beta_{j}+\gamma_{k}$, where $M_{i j}$ denotes the first age group $(i=25-29, \ldots, 85$ $+)$ in the $j$ period $(j=2003,2004, \ldots$ 2012) of expected incidence; $D_{i j}$ represents the number of people in the $i$ age group in the $j$ period; $P_{i j}$ corresponds to the population in the $i$ age group in the $j$ period; $\alpha_{i}$ denotes the age effect indicating the risk of population in the $i$ age group; $\beta_{j}$ refers to the period effect indicating the risk of population in the $j$ period; $\gamma_{k}$ stands for the cohort effect signifying the $k$ th $(k=i+j-1, k=1,2, \ldots, 14)$ incidence risk of birth cohort population; $\mu$ is the intercept.

Given the collinearity among three independent variables (age, period, and birth cohort), this study used the maximum likelihood estimation model and Epi in $R$ software ver. 3.5.0 (http://www.biostat.ku.dk/bxc/Epi) created by Carstensen to estimate the main parameters $\alpha i, \beta j$, and $\gamma_{k}$, thus resolving the collinearity of the APC model. ${ }^{17}$ In the maximum likelihood estimation model, the model with the highest degree of goodness of fit and lowest deviance value was selected as the best fitting model estimated using the posterior deviance test. ${ }^{18} P$-values $<0.05$ were considered statistically significant.

\section{Results}

A total of 4111 new cases of gastric cancer were diagnosed (3180 men and 931 women) in Changle in 2003-2012. The crude incidence rate of gastric cancer in Changle was 106.9 per 100,000 among men and 33.8 per 100,000 among women. The age-standardized incidence rates of gastric cancer among women reached 82.4 per 100,000 and 23.0 per 100,000 among men.

\section{Incidence Trends}

A steady downward trend was observed among men, with the incidence ranging from 96.15 per 100,000 in 2003 to 62.6 per 100,000 in 2012 (APC: $-5.1 \%, 95 \%$ CI:-6.9 to $-3.2 \%$ ). A similarly declining trend was observed among women with the incidence ranging from 34.5 per 100,000 to 15.7 per 100,000 (APC: $-5.7 \%, 95 \%$ CI:-9.3 to $-2.0 \%$ ) in the same period of diagnosis (Figure 1, Table 1). The declining trend appeared in 35-year-old and 45-year-old groups (by 10-year interval) among men from 2003 to 2012 (35-year-old group: APC $-8.4 \%$, 95\% CI:-13.3 to $-3.2 \%$; 45-year-old group: APC $-7.1 \%, 95 \%$ CI:-11.6 to $-2.3 \%$ ). The peak incidence was distributed among people over 65 years (Figure 2).

\section{Age-Period-Cohort Model}

By comparing the age, age-drift, age-period, age-cohort, and age-period-cohort models, the age-period-cohort model was selected as the model with the best fit due to the highest degree of goodness of fit and lowest deviance value for the incidence rate of gastric cancer in both men

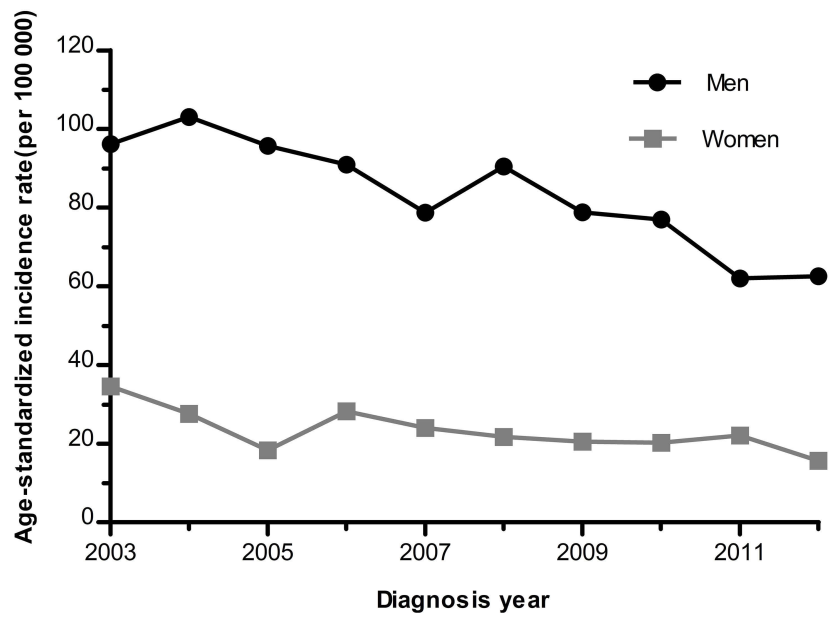

Figure I Age-standardized incidence rate of gastric cancer in Changle, 2003-20I2.

Table I Joinpoint Regression Analysis Of Trends In The Incidence Rate Of Gastric Cancer

\begin{tabular}{|l|l|l|l|l|}
\hline \multirow{2}{*}{ Gender } & \multicolumn{2}{|l|}{ ASR } & \multirow{2}{*}{ APC $(\mathbf{9 5} \% \mathbf{C l})$} & p-Value \\
\cline { 2 - 3 } & $\mathbf{2 0 0 3}$ & $\mathbf{2 0 1 2}$ & & \\
\hline Men & 96.2 & 62.6 & $-5.1(-6.9$ to -3.2$)$ & $<0.05$ \\
\hline Women & 34.5 & 15.7 & $-5.7(-9.3$ to -2.0$)$ & $<0.05$ \\
\hline
\end{tabular}

Abbreviations: ASR, age-standardized rate; APC, annual percent change. 

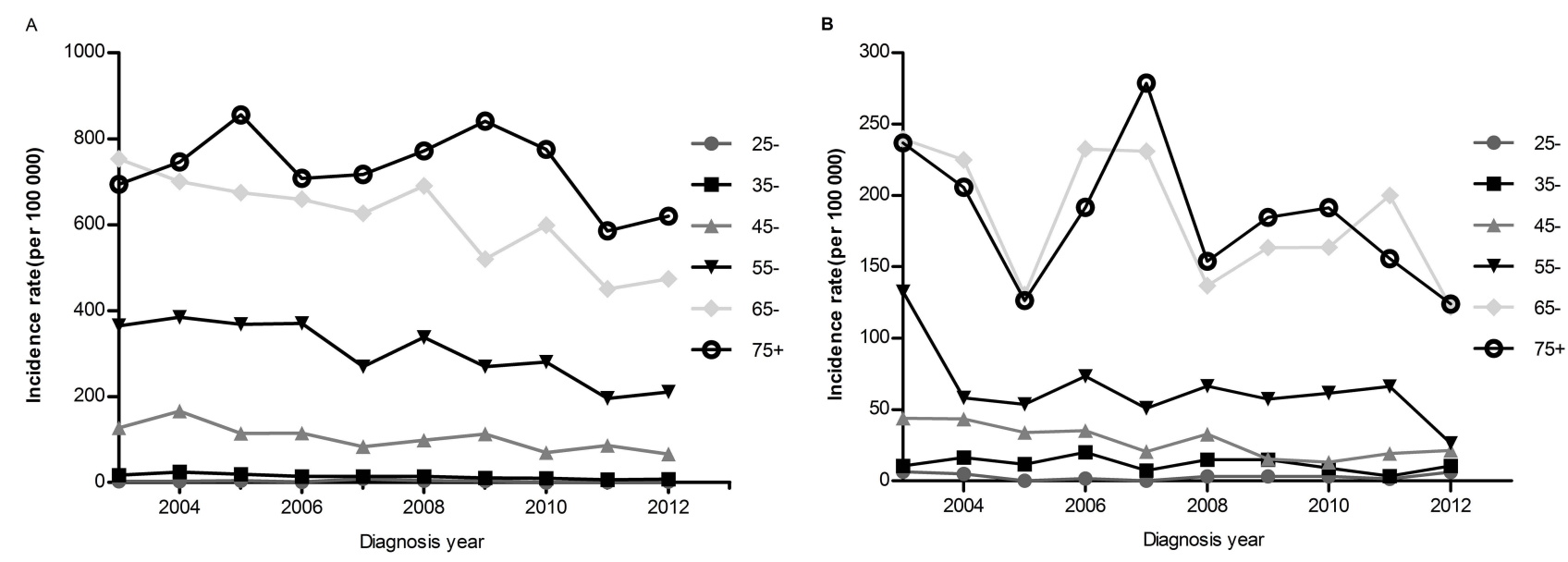

Figure 2 Age-specific incidence rate of gastric cancer by age groups among men (A) and women (B) in Changle, 2003-2012.

and women in Changle (Table 2). Figure $3 \mathrm{~A}$ and B showed two composite maps derived directly by R software; they reflected the effects of three variables: age, period, and birth cohort. We observed an increasing incidence rate of gastric cancer along with age in both men and women from the first curve (age effect) and a gradually decreasing incidence rate of gastric cancer in people later born in Changle from the second curve (birth cohort effect). The peak age for the incidence of gastric cancer was over 85 years in both men and women in Changle. The incidence rate of gastric cancer decreased gradually among men born after 1935 and among women born after 1940. Overall, from the third curve (period effect), the incidence rate of gastric cancer in both men and women in Changle showed a decreasing trend from 2003 to 2012.

\section{Discussion}

Our study showed the decreased incidence rates of gastric cancer in both men and women from 2003 to 2012. Our age-period-cohort models revealed that reduction in the incidence rate of gastric cancer was attributed to the decreased period of diagnosis and birth cohort effects. In addition, the incidence rate of gastric cancer gradually decreased in younger birth cohorts of women born after 1935 and men born after 1940.

\section{Age Effect}

This study showed that the incidence rate of gastric cancer increased with age, consistent with the results of previous studies in this area. ${ }^{19,20}$ Our results also showed that age exhibited a strong association with gastric cancer incidence

Table 2 Goodness Of Fit For The Age-Period-Cohort Models Of Gastric Cancer Incidence In Changle

\begin{tabular}{|c|c|c|c|c|c|c|}
\hline Gender & Model & Resid.Df & Resid.Dev & Df & Devianc & p-Value \\
\hline \multirow[t]{6}{*}{ Men } & Age & 124 & 4520.6 & & & \\
\hline & Age-drift & 123 & 2037.9 & I & 2482.7 & $<0.001$ \\
\hline & Age+cohort & 118 & 1483.6 & 5 & 554.3 & $<0.001$ \\
\hline & Age+period+cohort & 113 & 1447.9 & 5 & 35.7 & $<0.001$ \\
\hline & Age+period & 118 & 1977.5 & -5 & -529.6 & $<0.001$ \\
\hline & Age-drift & 123 & 2037.9 & -5 & -60.4 & $<0.001$ \\
\hline \multirow[t]{6}{*}{ Women } & Age & 124 & 2181.9 & & & \\
\hline & Age-drift & 123 & 1855.7 & I & 326.2 & $<0.001$ \\
\hline & Age+cohort & 118 & 1500.8 & 5 & 354.9 & $<0.001$ \\
\hline & Age+period+cohort & 113 & 1320.2 & 5 & 180.6 & $<0.001$ \\
\hline & Age+period & 118 & 1669.6 & -5 & -349.4 & $<0.001$ \\
\hline & Age-drift & 123 & 1855.7 & -5 & -186.10 & $<0.001$ \\
\hline
\end{tabular}

Abbreviation: Resid. DF, residual degrees of freedom. 

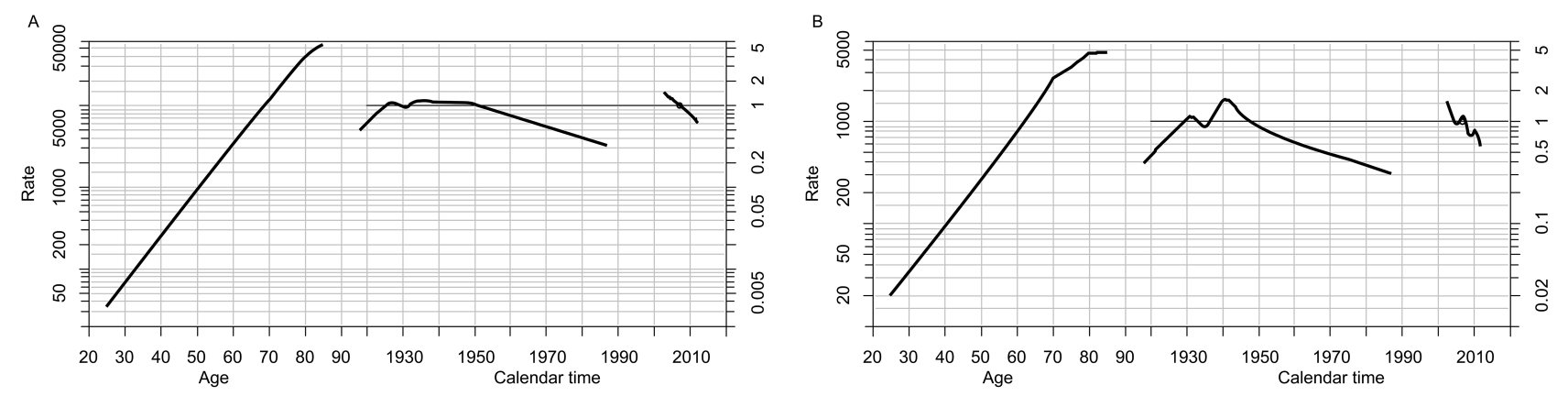

Figure 3 Fitted age-period-cohort model of the incidence rate of gastric cancer in Changle, from 2003 to 20I2. (A) Age, period, and cohort effects in men; (B) Age, period, and cohort effects in women.

in Changle. Age was regarded as the main factor in the ageperiod-cohort model analysis as it reflected consistent extrinsic factors, such as accumulative exposure of extrinsic environment, to a large degree. Age-related factors, such as accumulative exposures to carcinogens over time and accretion of mutations, are necessary factors of unregulated cell proliferation; this condition would lead to the occurrence of specific cancers. ${ }^{21}$ Our findings showed that the gastric cancer incidence decreased from 2003 to 2012. This result was verified in the joinpoint regression analysis.

\section{Period Of Diagnosis Effect}

The effect of period of diagnosis reflected the accumulation of most extrinsic factors, such as pollution or medical interventions, to a considerable extent because it affected each person at the same time in history. Similarly, we observed decreasing diagnosis effects on the incidence rate of gastric cancer. This finding could be explained by the improvement of medical health condition. After the discovery of the high incidence rate of gastric cancer in Changle in 1987, the Changle government implemented a series of measures, such as those focusing on drinking water and nutrition status, to improve the living environment of residents. In the past, $20 \%$ of residents drank surface water, and $80 \%$ drank well water. ${ }^{22}$ After the improvement, $80 \%$ of Changle residents moved into new houses, and all residents started to drink tap water. A 16year retrospective survey in this area showed that different types of drinking water were significantly related to gastric cancer mortality. ${ }^{23}$ The mortality rate of gastric cancer in drinking river water, well water and tap water group was 86.5 per $100,000,63.0$ per $100,000,29.8$ per 100,000 $(P<0.05)$. After the residents switched from drinking river and well water to tap water, the gastric cancer mortality significantly decreased by $59.1 \%$ (74.0/100,000 vs.
$30.3 / 100,000, P<0.05)$ and $56.4 \%(61.1 / 100,000$ vs.26.1/ $100,000, P<0.05)$, respectively. ${ }^{23}$

HP infection is regarded as the major risk factor of gastric cancer because it promotes precancerous lesions, such as atrophic gastritis and atypical hyperplasia. ${ }^{24}$ In 1994, a study on HP infection and gastric cancer in Changle revealed that the HP infection rate was significantly higher than that in Hong Kong (79.4\% [1744/2196] vs. $63.5 \%$ [548/863], $P<0.001){ }^{25}$ An epidemiological survey in the coastal areas of Fujian Province in 2012 showed that the infection rate of HP among Changle residents was $49.6 \%(111 / 224)$, which was significantly lower than that in $1994 \quad(49.6 \% \quad[111 / 224] \quad$ vs.79.4\% [1744/2196], $P<0.05) .{ }^{9}$ The decline in HP infection rate was attributed to improved sanitary conditions, nutritional status, and available HP antibiotics, thus reducing the gastric cancer incidence in Changle; this result was consistent with that of previous reports indicating a parallel variety between HP infection and gastric cancer incidence in developed countries. $^{10,26}$

\section{Cohort Effect}

The cohort effect reflected events, such as malnutrition of children or changing habits during the war, that affect generations. The decreased cohort effects on the incidence rate of gastric cancer in Changle residents among people born after 1940 might be attributed to the improvements in their lifestyle and habits.

Changle residents accustomed to eating fish sauce, which was a daily condiment favored previously by coastal residents. ${ }^{5}$ Fish sauce is a product of long-term fermentation and liquefaction from a variety of small sea fish added with salt; it contains a substantial amount of nitrosamines (secondary amines and amides). ${ }^{27}$ Several studies showed a correlation between fish sauce consumption and gastric 
cancer. $^{28-30}$ From 1991 to 1992 , Ye WM et al considered 14 towns from seven counties with high, medium, and low incidences of gastric cancer (Changle City as a representative of high incidence area) and observed a significantly positive association between fish sauce intake and mortality rate of gastric cancer. ${ }^{28}$ Chen $\mathrm{ZS}$ et al observed a $100 \%$ detection rate of nitrosamine in 17 portions of home-made fish sauce sold in Changle and four portions of fish sauce sold in other cities. ${ }^{29}$ Animal experiments indicated that fish sauce, under the action of nitrosamine ethyl creatine initiation factor, could promote carcinogenesis of mouse anterior gastric mucosal cells. ${ }^{30}$ In addition, fish sauce is a kind of salt-rich food. The World Cancer Research Fund/American Institute for Cancer Research concluded that "Salt, and also salt-preserved foods, are probable causes of gastric cancer". 31 A large cohort study in South Korea showed that people who eat salty foods face a high risk of stomach cancer. $^{32}$ Salt may increase the risk of gastric cancer by directly damaging the gastric mucosa, leading to gastritis or other stomach damage. ${ }^{33}$

Given the limited living conditions before the 1980s, Changle residents mainly fed on sweet potato shreds, which are easily mildewed because of damp climate in southeast coastal areas. An investigation in the 1990s showed that sweet potato silk at that time in Changle could culture aflatoxin, concomitant mycin, and blastomycin. The mycete infection rate in potato shreds reached $52 \%$ in the first year and $100 \%$ in the second year. ${ }^{34}$ Frequent consumption of salt and mildewy food is an unhealthy diet behavior. Since the 1980s, the lifestyle of residents in Changle has changed considerably. Most people started consuming grain as a staple food and replaced fish sauce with soy sauce. ${ }^{4,5}$ At the same time, people increased dietary intake of fresh fruit and vegetables, which are rich in antioxidant compounds, including $\beta$-carotene, vitamins $\mathrm{C}, \mathrm{E}$, and folate, and substances providing protection against gastric cancer. ${ }^{11,34}$

Another important factor contributing to the decreased gastric cancer prevalence was the change in the manner of food preservation. Many residents used refrigerators to preserve food since the $1980 \mathrm{~s}$; this situation was closely related to the declined incidence rate of gastric cancer. ${ }^{11,35}$ Researchers reported that the mortality rate of gastric cancer in Korea decreased thrice from 1979 to 2006; this result was significantly correlated with the increase in refrigerator utilization from 0.49 to $1.86 .^{36}$ A meta-analysis showed that increased risk of gastric cancer was associated with delayed use of household-refrigerated machinery. ${ }^{37}$ The potential reason might be the reduced decay and amounts of carcinogens, which could enter the body and increase the risk of gastric cancer, when keeping food in the fridge.

Many countries worldwide, including Japan with a previously high incidence of gastric cancer, showed a decreasing trend of incidence and mortality rate of gastric cancer in recent years. ${ }^{38-40}$ Similarly, a decreasing incidence rate of gastric cancer in recent years was also observed in Changle, which was considered a high incidence area of gastric cancer in this study. This phenomenon might be due to the reduced effect of period of diagnosis and birth cohort. This finding was inseparable with the evident improvements in the residents' lifestyle after the 1980s. We supposed that these improvements reduced the prevalence of gastric cancer in Changle, possibly providing a reference for other regions with a medium and high incidence of gastric cancer in the world to a certain extent.

This study features several strengths. First, this work was conducted in Changle, a high incidence area of gastric cancer and where a cancer registry database was established previously with a stable data quality and improved reliability of the analysis. Second, besides the function of traditional analysis methods, the APC model can further separate the independent roles of age, periods of diagnosis, and birth cohort in long-term registration data analysis. Third, the decline in the incidence rate of gastric cancer was dependent on the comprehensive preventive measures conducted in Changle, possibly providing a guiding significance for other regions. This work also suffered from several limitations. First, this study focused on the incidence rate of gastric cancer. Given the lack of uniform classification, we cannot conduct further studies on the histological types of gastric cancer. Second, this work was conducted in Changle county with a relatively limited population. The study results also possess limited power for generalization to other ethnicities and continents.

In conclusion, we identified the reduced effects of age, period of diagnosis, and birth cohort on the incidence rate of gastric cancer and recognized the trend of gastric cancer by sex, period of diagnosis, and birth cohort. The results might be due to a series of prevention and control measures to a large extent. Further health education and behavioral interventions are expected to prevent the occurrence and development of gastric cancer.

\section{Acknowledgments}

We are appreciative of all team staff for their contribution to the data collection and analysis and manuscript writing. This study was jointly supported by Science and Technology 
Program of Fujian Province, China (No. 2018Y2003) and Science and Technology Program of Fuzhou, Fujian Province, China (2015-S-163).

\section{Disclosure}

The authors report no conflicts of interest in this work.

\section{References}

1. Bray F, Ferlay J, Soerjomataram I, Siegel RL, Torre LA, Jemal A. Global cancer statistics 2018: GLOBOCAN estimates of incidence and mortality worldwide for 36 cancers in 185 countries. CA Cancer J Clin. 2018;68(6):394-424. doi:10.3322/caac.21492

2. Torre LA, Bray F, Siegel RL, Ferlay J, Lortet-Tieulent J, Jemal A. Global cancer statistics, 2012. CA Cancer J Clin. 2015;65(2):87-108.

3. He J, Chen WQ. Chinese Cancer Registry Annual Report, 2016. Beijing, China: Tsinghua University Press; 2017:72-110.

4. Chen W, Zheng R, Baade PD, et al. Cancer statistics in China, 2015. CA Cancer J Clin. 2016;66(2):115-132. doi:10.3322/caac.21338

5. Zheng TR, Chen ZC, Xiao JR, Chen JS. Review and prospect of high gastric cancer discovery site in Changle City. Chin Cancer. 2005; 14:312-314.

6. Zheng XW. Fujian Cancer Registry Annual Report, China, 2016. Fuzhou, China: Fujian Science and Technology Publishing House; 2018:66.

7. Sitarz R, Skierucha M, Mielko J, Offerhaus GJA, Maciejewski R, Polkowski WP. Gastric cancer: epidemiology, prevention, classification, and treatment. Cancer Manag Res. 2018;10:239-248. doi:10.2147/CMAR.S149619

8. Zheng XW. Fujian Cancer Registry Annual Report, China, 2017. Fuzhou, China: Fujian Science and Technology Publishing House; 2018:74.

9. Li XQ, Zhneg KC, Lin SK. Sero-epidemiological investigation on PG, G-17 and H. pylori antibody of coastal residents in Fujian Province. Chin Modern Prev Med. 2014;42:1543-1546.

10. Axon A. Helicobacter pylori: what do we still need to know? J Clin Gastroenterol. 2006;40(1):15-19. doi:10.1097/01.mcg.0000190752.486 21.fa

11. Chen ZC, Chen JS, Zheng TR. Analysis on the effect of prevention and treatment of gastric cancer in Changle City. Chin Cancer 2000;9:540-541.

12. Chen JS, Wu JP, Chen XC. Incidence trend of gastric cancer in Changle from 1989 to 1994. Chin Cancer. 1997;6:12-13.

13. Ying MG, Chen JS, Xiao JR. An analysis of temporal trend with cancer incidence from 1988 to 2002 in Changle. Chin Cancer. 2005; 14:32-33.

14. Clayton D, Schifflers E. Models for temporal variation in cancer rates. I: age-period and age-cohort models. Stat Med. 1987;6 (4):449-467. doi:10.1002/sim.4780060405

15. Segi M, Fujisaku S. Cancer Mortality for Selected Sites in 24 Countries (1950-1957). Sendai: Tohoku University Press; 1960.

16. Bethesda MD Joinpoint Regression Program Version 3.5.3. National Cancer Institute. 2012. Available from: http://surveillance.cancer.gov/ joinpoint/. Accessed August 20, 2018

17. Carstensen B. Age-period-cohort models for the lexis diagram. Stat Med. 2007;26(15):3018-3045. doi:10.1002/sim.2764

18. Holford TR. The estimation of age, period and cohort effects for vital rates. Biometrics. 1983;39(2):311-324. doi:10.2307/2531004

19. Karimi P, Islami F, Anandasabapathy S, Freedman ND, Kamangar F. Gastric cancer: descriptive epidemiology, risk factors, screening, and prevention. Cancer Epidemiol Biomarkers Prev. 2014;23:700-713. doi:10.1158/1055-9965.EPI-13-1057
20. Bao PP, Zheng Y, Wu CX. Cancer incidence in urban Shanghai,1973-2010: an updated trend and age-period-cohort effects. BMC Cancer. 2016;16:284.

21. Peto R, Parish SE, Gray RG. There is no such thing as ageing, and cancer is not related to it. IARC Sci Publ. 1985;58:43-53.

22. Yi YN, Lin TB, Chen ZC. Preliminary study on the pathogenic factors of gastric cancer in Changle County, Fujian Province. Chin Fujian Med J. 1987;9(3):23-25.

23. Wang ZQ, Chen Y, Lin YC. Study on drinking water type, water improvement and gastric cancer mortality in Changle County. Chin J Public Health. 1997;16:6-7.

24. Eslick GD. Helicobacter pylori infection causes gastric cancer? A review of the epidemiological, meta-analytic, and experimental evidence. World J Gastroenterol. 2006;12(19):2991-2999. doi:10.3748/ wjg.v12.i19.2991

25. Wang ZY, Lin ZX, Gao Z. Helicobacter pylori and gastric cancer. Chin J Gastroenterol. 1997;2:131-132.

26. Kelley JR, Duggan JM. Gastric cancer epidemiology and risk factors. $J$ Clin Epidemiol. 2003;56(1):1-9. doi:10.1016/S0895-4356(02)005 34-6

27. Zheng KC, Lin SG, Zhong WL. Survey on health condition and dietary custom in the residents of high and medium prevalence of gastric cancer in Fujian, China. Chin Strait J Prev Med. 2010;16::47.

28. Ye WM, Yi YN, Zhou TS. Epidemiological study on the relationship between edible fish sauce and mortality of gastric cancer in Fujian Province. Chin J Publ Heal. 1997;13:188-190.

29. Chen ZS, Yu L, Chen Y. Study on N-nitrosamines in fish sauce from high incidence area of gastric cancer. Chin J Cancer. 1988;7:81.

30. Cai HY, Huang KW, Jiao XR. Study on the effect of fish sauce on carcinogenesis and DNA synthesis of mouse foregastric epithelial cells. Chin J Cancer. 1984;3:166.

31. Wiseman M. The second World Cancer Research Fund/American Institute for Cancer Research expert report. Food, nutrition, physical activity, and the prevention of cancer: a global perspective. Proc Nutr Soc. 2008;67(3):253-256. doi:10.1017/S002966510800712X

32. Kim J, Park S, Nam BH. Gastric cancer and salt preference: a population-based cohort study in Korea. Am J Clin Nutr. 2010;91 (5):1289-1293. doi:10.3945/ajcn.2009.28732

33. Tsugane S, Sasazuki S, Kobayashi M, Sasaki S. Salt and salted food intake and subsequent risk of gastric cancer among middle-aged Japanese men and women. Br J Cancer. 2004;90(1):128-134. doi:10.1038/sj.bjc.6601511

34. Nomura AM, Hankin JH, Kolonel LN, Wilkens LR, Goodman MT, Stemmermann GN. Case-control study of diet and other risk factors for gastric cancer in Hawaii (United States). Cancer Causes Control. 2003;14(6):547-558. doi:10.1023/A:1024887411846

35. Zhou WB, Wu H, Cai Q. Research progress on risk factors of gastric cancer. Chin J Prac Intern Med. 2014;34:415-418.

36. Park B, Shin A, Park SK, et al. Ecological study for refrigerator use, salt, vegetable, and fruit intakes, and gastric cancer. Cancer Causes Control. 2011;22(11):1497-1502. doi:10.1007/s10552-011-9823-7

37. Boeing H. Epidemiological research in stomach cancer: progress over the last ten years. J Cancer Res Clin Oncol. 1991;117(2):133-143. doi:10.1007/BF01613137

38. Fitzmaurice C, Dicker D, Pain A, et al. The global burden of cancer 2013. JAMA Oncol. 2015;1:505-527. doi:10.1001/jamaoncol.2015. 0735

39. Katanoda K, Matsuda T, Matsuda A, et al. An updated report of the trends in cancer incidence and mortality in Japan. Jpn J Clin Oncol. 2013;43(5):492-507. doi:10.1093/jjco/hyt038

40. Siegel RL, Miller KD, Jemal A. Cancer statistics, 2016. CA Cancer J Clin. 2016;66(1):7-30. doi:10.3322/caac.21332 


\section{Publish your work in this journal}

Cancer Management and Research is an international, peer-reviewed open access journal focusing on cancer research and the optimal use of preventative and integrated treatment interventions to achieve improved outcomes, enhanced survival and quality of life for the cancer patient.
The manuscript management system is completely online and includes a very quick and fair peer-review system, which is all easy to use. Visit http://www.dovepress.com/testimonials.php to read real quotes from published authors.

Submit your manuscript here: https://www.dovepress.com/cancer-management-and-research-journal 